\title{
BMJ Open Association between diffusion tensor imaging findings and domain-specific cognitive impairment in cerebral small vessel disease: a protocol for systematic review and meta-analysis
}

\author{
Yao Xie (1) , ${ }^{1}$ Le Xie, ${ }^{1}$ Fuliang Kang, ${ }^{2}$ Junlin Jiang, ${ }^{1}$ Ting Yao, ${ }^{1}$ Yingchen Li, ${ }^{1}$ \\ Guo Mao, ${ }^{3}$ Dahua $\mathrm{Wu}^{1}$
}

To cite: Xie Y, Xie L, Kang F, et al. Association between diffusion tensor imaging findings and domain-specific cognitive impairment in cerebral small vessel disease: a protocol for systematic review and meta-analysis. BMJ Open 2021;11:e049203. doi:10.1136/ bmjopen-2021-049203

- Prepublication history for this paper is available online. To view these files, please visit the journal online (http://dx.doi org/10.1136/bmjopen-2021049203).

Received 20 January 2021 Accepted 03 September 2021

Check for updates

(c) Author(s) (or their employer(s)) 2021. Re-use permitted under CC BY-NC. No commercial re-use. See rights and permissions. Published by BMJ.

${ }^{1}$ Department of Neurology, Hunan University of Chinese Medicine Integrated Chinese Medicine Affiliated Hospital, Changsha, Hunan, China

${ }^{2}$ Department of Imaging, Hunan University of Chinese Medicine Integrated Chinese Medicine Affiliated Hospital, Changsha, Hunan, China

${ }^{3}$ Office of Academic Research, Hunan University of Chinese

Medicine Integrated Chinese

Medicine Affiliated Hospital,

Changsha, Hunan, China

Correspondence to

Dr Dahua Wu;

547292206@qq.com

\section{ABSTRACT}

Introduction Cognitive impairment is the main clinical manifestation of cerebral small vessel disease (CSVD). However, the mechanism and structural damage in different domains of cognitive disorders are poorly understood. There is an urgent need to quantify the relation between diffusion tensor imaging (DTI) data and impaired cognitive testing in CSVD, which may help to find biomarkers for early diagnosis or treatment evaluation. We aim to summarise the understanding of association between DTI findings and domain-specific cognitive impairment.

Methods and analysis PubMed, EMBASE, Web of science, Cochrane library, Chinese National Knowledge Infrastructure Databases, Wanfang, SinoMed and VIP will be searched, from 1 January 1994 to 1 August 2021. The ClinicalTrials. gov and Chictr.org.cn records will also be searched to identify further potential studies. The included studies should report fractional anisotropy and/or and mean diffusivity/ apparent diffusion coefficient data for one or more individual regions of interest in DTI analysis. Meanwhile, cognitive testing scores are also needed. This systematic review will be reported according to the Preferred Reporting Items for Systematic Reviews and Meta-Analyses. The quality of cohort or case-control studies will be evaluated by the Newcastle-Ottawa Scale, and the cross-section studies will be evaluated by Agency for Healthcare Research and Quality scale. Meta-analysis, subgroup and sensitivity analyses, and publication bias will be all performed with Stata.

Ethics and dissemination Patients and the public will not be involved in this study. The existing data from published studies will be used. The findings from this research will be relevant information regarding the association of DTI metrics with cognitive disorder, which will be published in a peer-reviewed journal. If we need to amend this protocol, we will give the date of each amendment, describe the change and give the rationale. Changes will not be incorporated into the protocol.

PROSPERO registration number CRD42021226133.

\section{INTRODUCTION}

Cerebral small vessel disease (CSVD) denotes a range of clinical, imaging and pathological

\section{Strengths and limitations of this study}

- To our knowledge, this is the first meta-analysis research to explore the association between diffusion tensor imaging (DTI) findings and cognitive outcomes following cerebral small vessel disease (CSVD).

- The findings of this review and meta-analysis will contribute to understand pathology of different impaired cognitive domains in CSVD.

- The conducted meta-analysis will help to diagnostic performance of the technique in cognitive impairment of CSVD.

- Our research will help to explore new recognised biomarkers to monitor progression of disease, or evaluate the treatment efficacy, and it will be helpful to development in treating cognitive impairment of CSVD.

- The limitation will be the heterogeneity among included studies in image acquisition techniques, postprocessing image analysis methodologies and variability in the extracted DTI metrics.

syndromes resulting from various causes affecting the small arteries, arterioles, capillaries and venules in the brain. ${ }^{1}$ Cognitive disorder is one of the most common clinical features in CSVD. Moreover, CSVD is the most common cause of vascular cognitive impairment and contributes to up to $45 \%$ of dementias. ${ }^{2}$ The domain of cognitive impairment caused by CSVD may include the executive function, attention, working memory and psychomotor speed. ${ }^{3}$ However, the mechanism and structural damage of cognitive disorder in CSVD is still unknown.

There is a pressing need for better understanding of the pathological mechanism to early diagnosis or help to find effective treatments. The pathology of CSVD mainly lead to white matter (WM) lesions which can be 
detected by CT or MRI. WM is nerve fibres to interconnect neurons in the cerebral cortex or the deep structures. Therefore, if WM is disrupted, the damaged WM may cause the disconnection or network disruption in the brain. More and more evidence showed WM played an important role in cognitive decline and dementia. ${ }^{4}$ Bos $e t$ $a \tilde{l}$ summarised that WM hyperintensities were associated with an increased risk of dementia in 3913 participants from 11 studies. Rensma et at also found higher dementia rates in CSVD (1709 dementia events in 16548 CSVD individuals) from 28 studies. So studies are warranted to further determine the role of markers of CSVD in cognitive impairment.

Neuroimaging is the crucial implement to detect brain injury of CSVD, especially the diffusion tensor imaging (DTI). DTI is a kind of MRI technology to detect microscopic structural changes in WM and evaluate the structural integrity of WM fibres. In DTI analysis, the lower fractional anisotropy (FA) and higher mean diffusivity (MD) generally reflect lower microstructural connectivity. Previous studies found some WM changes in DTI were associated with different domain-specific cognitive disorders. The Radboud University Nijmegen Diffusion tensor and Magnetic resonance imaging Cohort (RUN DMC) Study found the MD was associated with the verbal memory performance, psychomotor speed and attention in 499 individuals with CSVD. ${ }^{7}$ Tuladhar et $a l^{8}$ reported DTI findings in the genu and splenium of corpus callosum showed evident relation with executive functions, and the cingulum bundle was also associated with verbal memory performance. Duering et at reported frontal interhemispheric and thalamic projection fibre tracts measured by DTI were related to processing speed.

However, the relationship between DTI and cognitive outcomes in CSVD is currently unclear. The different cognitive domains were examined separately in different studies, as were the DTI metrics from different regions of interest (ROIs). To our knowledge, there are no metaanalysis research to explore the association between DTI findings and cognitive outcomes following CSVD. The purpose of this study protocol is to provide a clear methodology to review the relation between DTI and cognitive impairment in CSVD.

\section{Objective}

We perform the meta-analysis to summary knowledge and understanding of the association between DTI findings and domain-specific cognitive impairment in CSVD.

\section{Review questions}

Which cognitive functions are impaired in CSVD? Which microscopic structure changes measured by DTI is associated with different impaired cognitive domains?

\section{METHODS}

This protocol was abided by Preferred Reporting Items for Systematic Reviews and Meta-Analyses (PRISMA)
Protocols. $^{10}$ This research will be according to the PRISMA statement and guidance. ${ }^{11}$

\section{Inclusion and exclusion criteria}

The following criteria are applied to select studies ${ }^{1}$ : These studies examined adults ( $\geq 18$ years) who underwent brain imaging to determine CSVD by MRI or CT. ${ }^{122}$ Some of the participants were confirmed to have cognitive impairment ${ }^{1}$ with at least one cognitive domain affected. ${ }^{3}$ FA and /or MD/apparent diffusion coefficient data were reported for one or more ROIs in DTI analysis. ${ }^{4}$ Cognitive testing scores were reported. ${ }^{5}$ Correlations between cognitive testing scores and DTI values were reported (Pearson's $r$, Spearman's r or exact $\mathrm{p}$ values provided). ${ }^{6}$ The studies followed a cohort or case-control or cross-sectional design. On the other hand, we will exclude studies if ${ }^{1}$ : CSVD occurred in a context of immune-mediated or neurodegenerative disorders (eg, multiple sclerosis or Parkinson's disease). ${ }^{2}$ The cognitive disorder was mainly caused by other diseases (eg, Alzheimer's disease or Lewy body dementia). ${ }^{3}$ Reviews, case studies and studies with very small samples $(\mathrm{N} \leq 5) .{ }^{4}$ DTI data or scores can not be extracted, calculated or provided by the study authors. ${ }^{5}$ Other studies presented research data that overlap with already included studies.

\section{Search strategy for identification of studies}

The following databases will systematically screened for literatures: PubMed, EMBASE, Web of Science, Cochrane library, Chinese National Knowledge Infrastructure Databases, Wanfang, SinoMed and Chongqing Chinese Science and Technology Periodical Database (VIP), from 1 January 1994 to 1 August 2021. The ClinicalTrials.gov and Chictr.org.cn records will also be searched to identify further potential studies. If some researchers are interested in our study, we will be happy to share relevant databases or papers.

The search strategy used the following terms: (1) "cerebral small vessel disease" or "small vessel disease" or "lacunar" or "subcortical infarct" or "subcortical stroke" or "microinfarct" or "subcortical lesion" or "cerebrovascular disorder" or "Virchow-Robin space" or "perivascular space" or "leukoaraiosis" or "leuko-araiosis" or "white matter lesion" or "white matter hyperintensities" or "white matter hyperintensity" or "cerebral microbleed" or "micro haemorrhage" or "micro hemorrhage", (2) "cognitive impairment" or "cognitive disorder" or "dementia" or "cognition" or "executive" or "executive function" or "memory" or "attention" or "psychomotor speed" or "processing speed" or "learning" or "language" or "social cognition" or "praxis" or "gnosis" or "visual perception", (3) "diffusion tensor imaging" or "diffusion imaging" or "fractional anisotropy" or "mean diffusivity" or "apparent diffusion coefficient". The suggested search syntax on PubMed is summarised in table 1 . The flow chart is illustrated in figure 1 . 
Table 1 Search Syntax in PubMed

\begin{tabular}{ll}
\hline Database & Search Syntax \\
\hline PubMed & \#1: $(((((((((((((((((($ cerebral small vessel disease(Title/Abstract))) OR (CSVD(Title/Abstract))) OR (small vessel \\
& disease(Title/Abstract))) OR (SVD(Title/Abstract))) OR (lacunar(Title/Abstract))) OR (subcortical infarct(Title/ \\
& Abstract))) OR (subcortical stroke(Title/Abstract))) OR (microinfarct(Title/Abstract))) OR (subcortical lesion(Title/ \\
& Abstract))) OR (cerebrovascular disorder(Title/Abstract))) OR (Virchow-Robin space(Title/Abstract))) OR \\
& (perivascular space(Title/Abstract))) OR (leukoaraiosis(Title/Abstract))) OR (leuko-araiosis(Title/Abstract))) \\
& OR (white matter lesion(Title/Abstract))) OR (white matter hyperintensities(Title/Abstract))) OR (white matter \\
& hyperintensity(Title/Abstract))) OR (WMH(Title/Abstract))) OR (cerebral microbleed(Title/Abstract))) OR (micro \\
& hemorrhage(Title/Abstract)) \\
& \#2: ((((((((((((((cognitive impairment(Title/Abstract)) OR (cognitive disorder(Title/Abstract))) OR (dementia(Title/ \\
& Abstract))) OR (cognition(Title/Abstract))) OR (executive(Title/Abstract))) OR (executive function(Title/Abstract))) \\
& OR (memory(Title/Abstract))) OR (attention(Title/Abstract))) OR (psychomotor speed(Title/Abstract))) OR \\
& (processing speed(Title/Abstract))) OR (learning(Title/Abstract))) OR (language(Title/Abstract))) OR (social \\
& cognition(Title/Abstract))) OR (praxis(Title/Abstract))) OR (gnosis(Title/Abstract))) OR (visual perception(Title/ \\
& Abstract)) \\
& \#3: (((((((diffusion tensor imaging(Title/Abstract)) OR (DTI(Title/Abstract))) OR (fractional anisotropy(Title/Abstract))) \\
& OR (mean diffusivity(Title/Abstract))) OR (apparent diffusion coefficient(Title/Abstract))) OR (FA(Title/Abstract))) OR \\
& (MD(Title/Abstract))) OR (ADC(Title/Abstract))) OR (diffusion imaging(Title/Abstract)) \\
& \#4: \#1 AND \#2 AND \#3
\end{tabular}

\section{Study selection for inclusion in the review}

Literature search results will be imported into NoteExpress software (V.3.0) and duplicate records will be deleted. One researcher (YX) will screen titles of articles to exclude the obviously irrelevant studies, and the full text of relevant studies will be obtained. All abstracts and full-text articles of potentially relevant articles will be independently screened by two researchers (YX and LX). Any disagreements will be resolved by discussion or decided by a third researcher (FK). When there are no available data, investigators of studies should be contacted by email. In the case of multiple publications from the same study, we will include the one which has the most up-todate or comprehensive information.

\section{Data extraction and management}

The following data will be extracted: publication, general information, study design, participant characteristics, control, comorbidity, type of CSVD, cognitive severity, DTI metrics, cognitive testing, brand of scanner, MRI magnet strength, method(s) of analysis, duration of follow-up, etc. Cognitive testing will be categorised into seven cognitive domains according to previous studies ${ }^{131}$ : general cognition, ${ }^{2}$ memory, ${ }^{3}$ attention, processing speed and working memory, ${ }^{4}$ executive function, ${ }^{5}$ verbal skills, ${ }^{6}$ concept formation and reasoning, ${ }^{7}$ construction and motor performance. The data will be evaluated and extracted independently by two researchers (YX and LX).

\section{Quality assessment}

Newcastle-Ottawa Scale (NOS) will be used to evaluate the quality of cohort or case-control studies. ${ }^{14}$ It contains eight items, mainly covering selection, comparability, exposure and outcome. The full score of NOS is 9 stars. The quality of the studies will be graded quality as good ( $\geq 7$ stars), fair ( $4-6$ stars) or poor ( $<4$ stars). The quality of the cross-sectional studies will be assessed using the
Agency for Healthcare Research and Quality scale, ${ }^{15}$ which contains 11 items. Study quality will be assessed as follows: low quality ( $<3$ score), moderate quality (4-7 score) and high quality (8-11 score).

\section{Statistical analysis}

Pearson's $r$ will be applied to assess the relationship between the DTI metrics for each ROI and individual cognitive domain. Spearman's $r$ will be transformed with the equation $\left(r=2 \sin \left[r_{s} \pi / 6\right]\right) .{ }^{16}$ Then, summary $r$ will be calculated with fisher's Z, which is transformed with Pearson's r. ${ }^{17}$ Effect sizes (summary r) of 0.1, 0.3, 0.5 and 0.7 correspond to small, moderate, strong and very strong effects. ${ }^{18}$

The following subgroups analyses are planned: aetiology (arteriosclerosis vs inheritance vs others), study type (cohort vs case-control vs cross-sectional studies), magnet strength $(1.5 \mathrm{~T}$ vs $>1.5 \mathrm{~T})$ and sample size $(\leq 30$ vs $>30$ ).

The random effects model (Der Simonian and Laird method ${ }^{19}$ will be applied in all meta-analyse, because the clinical and methodological condition differs to some extent in included studies. A random effects model is more conservative. Q-test has poor power to analyse heterogeneity in the situation of few studies. ${ }^{20}$ Heterogeneity among the included studies will be assessed by $\mathrm{I}^{2}$. According to Cochrane Handbook for Systematic Reviews of Interventions (V.5.1.0), the degree of heterogeneity is defined by $\mathrm{I}^{2}$ value: might not be important $(0 \%-40 \%)$, moderate heterogeneity $(30 \%-60 \%)$, substantial heterogeneity $(50 \%-90 \%)$, considerable heterogeneity $(75 \%-100 \%)$.

Publication bias will be assessed by the funnel plot and Egger's test. The $p<0.05$ is considered statistically significant. Sensitivity analyses will also be undertaken to analyse the stability of results in meta-analysis, such as according 


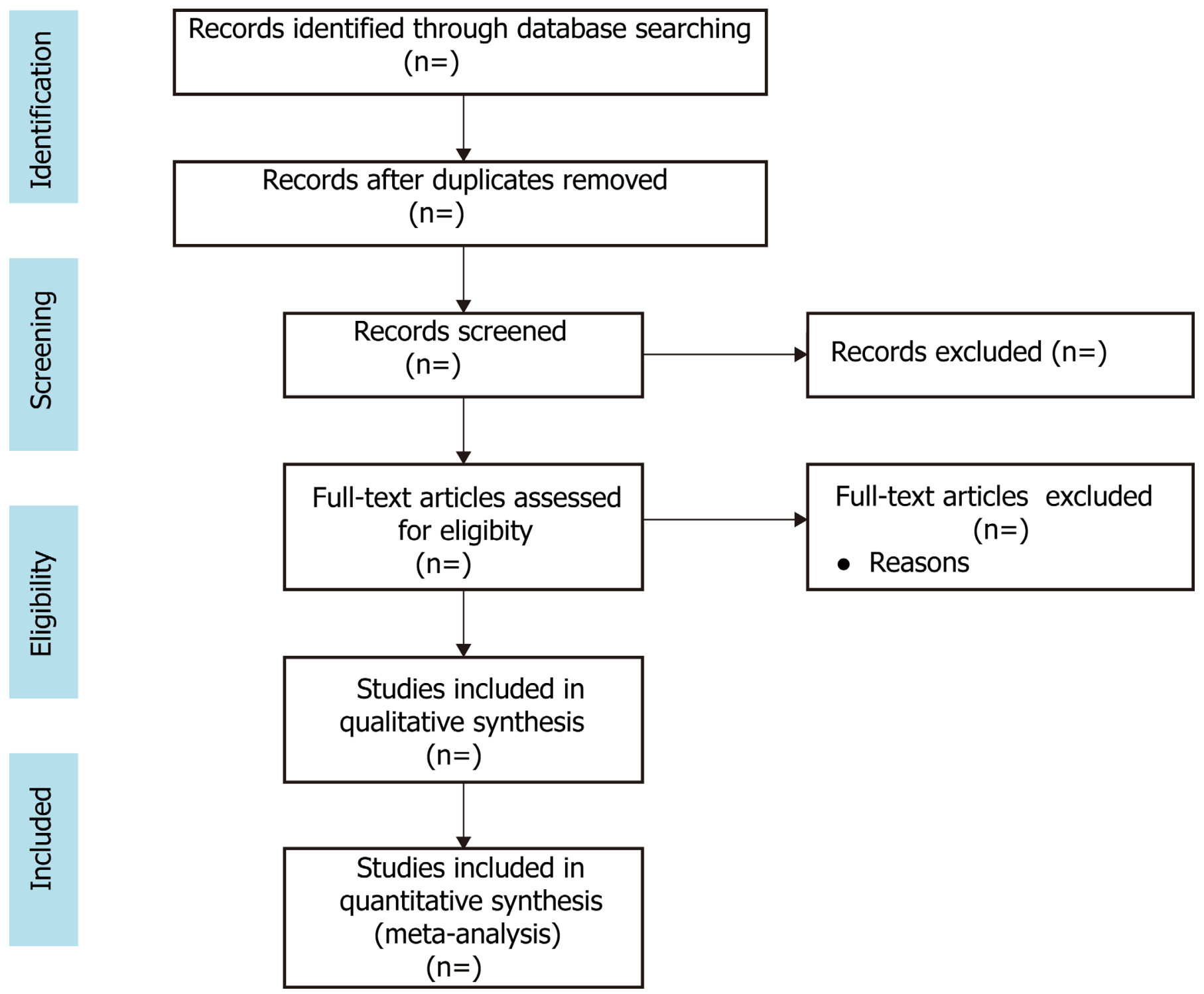

Figure 1 Flow diagram for search strategy.

to the quality of studies. All analyses were performed with Stata V.15.1.

If quantitative synthesis is not appropriate, a systematic narrative synthesis will be used, we will provide information in tables to summarise and explain the characteristics and findings from the included studies.

\section{Patient and public involvement}

The research will use existing data from published studies. Patients and the public will not be involved in the design of this study.

\section{DISCUSSION}

After finished above research content, we will get the impaired cognitive domains leaded by CSVD. Moreover, the disrupted WM microscopic structure associated with different impaired cognitive domains will be further known, which could encourage to improve diagnosis and treatment.
In the past decade, DTI has been increasingly used for the evaluation of CSVD patients, because it is the high sensitivity in detecting cerebral tissue damage. However, up to now, no meta-analysis has been carried out to quantify the relation between DTI data and impaired cognitive testing in CSVD. Actually, many studies had been completed for the relation. They all demonstrated that low FA and high MD in multiple regions of the WM were associated with lower scores on cognitive performance. $^{82122}$ There are variations in the results of different studies, because of the difference in ROIs, the study type, sample size, the subject and so on. It is of great significance to diagnosis and treatment of cognitive impairment in CSVD by studying the DTI associated with cognition.

Currently, little is known about neural basis of impaired cognitive function. Subsequently, there are few diseasespecific treatments for cognitive impairment of CSVD. However, the disease can be prevented in the early stage. ${ }^{1}$ So we should explore the biomarker to early diagnose the 
cognitive impairment in CSVD. Our research will help to understand pathology and develop new recognised biomarkers to diagnosis, monitor progression of disease or evaluate the treatment efficacy.

Contributors The idea of this research was proposed by YX. The literature search, article screen, data extraction and analysis will be contributed by YX, LX and FK. This protocol drafting was undertaken by YX, JJ, TY, YL and GM. YX and DW revised the manuscript for intellectual content. All authors approved the final version of the manuscript.

Funding This research was supported by Open Fund for First-class Disciplines of Integrated Traditional Chinese and Western Medicine (No.2020ZXYJH10), Hunan Academy of Traditional Chinese Medicine Foundation (No.201920), Foundation of China Center for Evidence Based Traditional Chinese Medicine (No.2019XZZX-NB004), The National Key Research and Development Project (No.2018YFC1704904). Those provided the team and fund for the systematic review.

\section{Competing interests None declared.}

Patient and public involvement Patients and/or the public were not involved in the design, or conduct, or reporting, or dissemination plans of this research.

Patient consent for publication Not applicable.

Provenance and peer review Not commissioned; externally peer reviewed.

Open access This is an open access article distributed in accordance with the Creative Commons Attribution Non Commercial (CC BY-NC 4.0) license, which permits others to distribute, remix, adapt, build upon this work non-commercially, and license their derivative works on different terms, provided the original work is properly cited, appropriate credit is given, any changes made indicated, and the use is non-commercial. See: http://creativecommons.org/licenses/by-nc/4.0/.

ORCID iD

Yao Xie http://orcid.org/0000-0002-9885-3858

\section{REFERENCES}

1 Geriatric Neurology Group, Chinese Society of Geriatrics, Clinical Practice Guideline for Cognitive Impairment of Cerebral Small Vessel Disease Writing Group. Clinical practice guideline for cognitive impairment of cerebral small vessel disease. Aging Med 2019;2:64-73.

2 Gorelick PB, Scuteri A, Black SE, et al. Vascular contributions to cognitive impairment and dementia: a statement for healthcare professionals from the American heart association/american stroke association. Stroke 2011;42:2672-713.

3 Pasi M, van Uden IWM, Tuladhar AM, et al. White matter microstructural damage on diffusion tensor imaging in cerebral small vessel disease: clinical consequences. Stroke 2016;47:1679-84.
4 Prins ND, Scheltens P. White matter hyperintensities, cognitive impairment and dementia: an update. Nat Rev Neurol 2015;11:157-65.

5 Bos D, Wolters FJ, Darweesh SKL, et al. Cerebral small vessel disease and the risk of dementia: a systematic review and metaanalysis of population-based evidence. Alzheimers Dement 2018;14:1482-92.

6 Rensma SP, van Sloten TT, Launer LJ, et al. Cerebral small vessel disease and risk of incident stroke, dementia and depression, and all-cause mortality: a systematic review and meta-analysis. Neurosci Biobehav Rev 2018;90:164-73.

7 van Norden AGW, van Uden IWM, de Laat KF, et al. Cognitive function in small vessel disease: the additional value of diffusion tensor imaging to conventional magnetic resonance imaging: the run DMC study. J Alzheimers Dis 2012;32:667-76.

8 Tuladhar AM, van Norden AGW, de Laat KF, et al. White matter integrity in small vessel disease is related to cognition. Neuroimage 2015;7:518-24.

9 Duering M, Gesierich B, Seiler S, et al. Strategic white matter tracts for processing speed deficits in age-related small vessel disease. Neurology 2014;82:1946-50.

10 Shamseer L, Moher D, Clarke M, et al. Preferred reporting items for systematic review and meta-analysis protocols (PRISMA-P) 2015: elaboration and explanation. BMJ 2015;350:g7647.

11 Moher D, Liberati A, Tetzlaff J, et al. Preferred reporting items for systematic reviews and meta-analyses: the PRISMA statement. PLoS Med 2009;6:e1000097.

12 Wardlaw JM, Smith EE, Biessels GJ, et al. Neuroimaging standards for research into small vessel disease and its contribution to ageing and neurodegeneration. Lancet Neurol 2013;12:822-38.

13 Lezak M, Howieson D, Bigler E. Neuropsychological assessment. 5th edition[M]. New York: Oxford University Press, 2012.

14 Wells G. The Newcastle-Ottawa scale (NOS) for assessing the quality of nonrandomised studies in meta-analysis, 2004. Available: http:// www.ohri.ca/programs/clinical_epidemiology.oxford

15 Rostom A, Dubé C, Cranney A. Celiac disease. Rockville (MD): Agency for Healthcare Research and Quality (US), 2004. https:// www.ncbi.nlm.nih.gov/books/NBK35156/

16 Rupinski MT, Dunlap WP. Approximating Pearson Product-Moment Correlations from Kendall's Tau and Spearman's Rho. Educ Psychol Meas 1996;56:419-29.

17 Borenstein M, Hedges L V, Higgins JPT, et al. An introduction to meta-analysis. Hoboken: John Wiley \& Sons, 2009.

18 Rosenthal JA. Qualitative descriptors of strength of association and effect size. J Soc Serv Res 1996;21:37-59.

19 Higgins JPT, Thompson SG. Quantifying heterogeneity in a metaanalysis. Stat Med 2002;21:1539-58.

20 DerSimonian R, Laird N, Nan L. Meta-analysis in clinical trials. Control Clin Trials 1986;7:177-88.

21 Chen HJ, Gao YQ, Che CH. Diffusion tensor imaging with tractbased spatial statistics reveals white matter abnormalities in patients with vascular cognitive impairment. Front Neuroanat 2018;26:53.

22 Lin L, Xue Y, Duan Q, et al. Microstructural white matter abnormalities and cognitive dysfunction in subcortical ischemic vascular disease: an atlas-based diffusion tensor analysis study. $J$ Mol Neurosci 2015;56:363-70. 